\title{
Perfil de recém-nascidos submetidos à cirurgia cardíaca em hospital privado do Município de São Paulo*
}

\author{
PROFILE OF NEWBORNS WHO UNDERWENT CARDIAC SURGERY IN A \\ PRIVATE HOSPITAL IN THE CITY OF SÃO PAULO
}

\author{
PERFIL DE LOS RECIÉN NACIDOS SOMETIDOS A CIRUGÍA CARDIACA EN UN \\ HOSPITAL PRIVADO DEL MUNICIPIO DE SAO PAULO
}

\author{
Mariana Bueno', Amélia Fumiko Kimura²
}

\begin{abstract}
RESUMO
Estudo transversal cujo objetivo foi descrever o perfil dos recém-nascidos submetidos à cirurgia cardíaca em um hospital privado, referência em cirurgia cardíaca neonatal, no município de São Paulo. Foram analisados prontuários de 30 pacientes internados entre julho de 2001 a dezembro de 2005. Foram incluídos neonatos com idade $\geq 35$ semanas, com anomalias cardíacas. Excluíram-se os neonatos com óbito no $1 .^{\circ}$ pós-operatório (OP) e com outras malformações sistêmicas. A maioria dos RNs nasceu a termo, com peso $\geq$ a $2.500 \mathrm{~g}$ e submetidos à cirurgia na primeira semana de vida. Foram identificados 24 diferentes diagnósticos de cardiopatias e 14 diferentes intervenções cirúrgicas. No $1 .^{\circ} \mathrm{PO}$, todos os neonatos estavam com dispositivos para monitorar os parâmetros vitais ou como via de acesso para tratamento e suporte de vida, mostrando não só a complexidade do tratamento, como a necessidade de planejamento e organização do serviço na implementação da assistência a estes pacientes.
\end{abstract}

\section{DESCRITORES}

Recém-nascido.

Cardiopatias congênitas.

Cirurgia torácica.

Cuidados de enfermagem.

\begin{abstract}
The aim of this cross-sectional study was to characterize newborns that underwent cardiac surgery in a private hospital, a reference center in neonatal cardiac surgery, in the city of São Paulo. Data were collected from medical reports from July, 2001 to December, 2005. Newborns with gestational age of more than 35 weeks were included in the study. Newborns that died on the first 48 postoperative hours and with other malformations were excluded. Most of the neonates were term, weighted 2,500 grams or more, and underwent surgery in the first week of life. There were 24 different diagnoses identified and 14 different types of surgery performed. In the first post-operative day, all newborns had invasive devices for vital signs monitoring or for treatment and life support, which shows not only the complexity of the treatment but the need for planning and organizing health services in order to provide adequate care for these patients.
\end{abstract}

\section{KEY WORDS}

Infant, newborn.

Heart defects, congenital.

Thoracic surgery.

Nursing care.

\section{RESUMEN}

El objetivo en este estudio transversal fue caracterizar a los recién nacidos sometidos a cirugía cardiaca en un hospital privado del municipio de Sao Paulo. Los dados fueron obtenidos de las historias clínicas de los recién nacidos sometidos a cirugía entre julio de 2001 y diciembre del 2005. Los recién nacidos con 35 semanas o más de gestación fueron incluidos y excluidos los recién nacidos con óbito en el 1er dia de post-operatorio ( $\mathrm{PO}$ ) y con otras malformaciones sistémicas. La mayoría de los RNs nació a término, con peso ${ }^{3}$ a 2.500 g e sometidos a la cirugía en la primera semana de vida. Fueron identificados 24 diferentes diagnósticos de cardiopatías y 14 diferentes intervenciones quirúrgicas. En el 1er dia de $\mathrm{PO}$, todos los neonatos estaban con dispositivos para monitorear los parámetros vitales o como vía de acceso para el tratamiento y soporte de vida, mostrando no sólo la complejidad del tratamiento, sino también la necesidad de planificación y organización del servicio en la implementación de la asistencia a estos pacientes.

\section{DESCRIPTORES}

Recién nacido.

Cardiopatias congénitas.

Cirugía torácica.

Atención de enfermería.

\footnotetext{
* Artigo extraído da dissertação "Dor e analgesia em recém-nascidos submetidos a cirurgia cardíaca", Universidade de São Paulo (USP), 2006. ${ }^{1}$ Enfermeira do Núcleo de Apoio à Pesquisa Clínica do Hospital das clínicas da Faculdade de Medicina da USP Especialista em Enfermagem Neonatal, Mestranda em Enfermagem Neonatal pela Escola de Enfermagem da USP (EE/USP). São Paulo, SP, Brasil. maribueno@hotmail.com ${ }^{2}$ Enfermeira, Professora Doutora do Departamento de Enfermagem Materno Infantil e Psiquiátrica da EE/USP. São Paulo, SP, Brasil.
} 


\section{INTRODUÇÃO}

Os avanços tecnológicos ocorridos nas últimas décadas possibilitaram a identificação das malformações cardíacas e o aprimoramento das técnicas cirúrgicas, dos cuidados pós-operatórios ( $\mathrm{PO}$ ), da terapia medicamentosa e do manejo da ventilação pulmonar mecânica. Além disso, houve avanços no conhecimento da fisiopatologia cardíaca e de suas repercussões, o que contribuiu para afastar as complicações PO, reduzindo as taxas de morbidade e aumentando as taxas de sobrevida, em longo prazo, de pacientes neonatais e pediátricos portadores de anomalias cardíacas congênitas ${ }^{(1-4)}$. Desse modo, percebe-se que as cardiopatias congênitas acometem entre oito e dez neonatos em 1.000 nascidos vivos ${ }^{(5-8)}$.

A escolha da técnica cirúrgica, bem como do caráter paliativo ou corretivo da intervenção são definidas, sobretudo, com base na anatomia cardíaca e no quadro clínico do recém-nascido (RN). Recomenda-se que os procedimentos cirúrgicos sejam realizados, preferencialmente, no período neonatal e, se possível, por reparo definitivo, incluindo das lesões complexas, sendo o principal objetivo minimizar os efeitos secundários de lesões não operadas, a exemplo da insuficiência cardíaca congestiva crônica que traz como conseqüência retardo no desenvolvimento, infecções recorrentes, alterações vasculares pulmonares irreversíveis, atraso no desenvolvimento cognitivo e déficit neurológico focal|(6,9).

Além da intervenção cirúrgica em si, a assistência PO é de fundamental importância para atenuar ou evitar complicações e auxiliar no restabelecimento do RN contribuindo, assim, para o sucesso do procedimento. As ações necessárias ao adequado cuidado PO incluem moni-torização dos parâmetros hemodinâmicos, ventilatórios, manutenção de equilíbrio hidroeletrolítico e balanço hídrico, cuidado com sondas, drenos e cateteres, administração de drogas vasoativas, antibióticos, hemoderivados, analgésicos e sedativos dentre outros ${ }^{(1,4,10)}$.

Portanto, recursos humanos, materiais e logísticos são necessários para garantir um atendimento PO de qualidade.

Conhecer o perfil da população com a qual se trabaIha favorece a elaboração do plano assistencial pelo enfermeiro e o provimento de uma assistência adequada pela equipe de enfermagem. Assim, é possível contribuir na minimização das complicações e proporcionar condições favoráveis ao atendimento PO e ao restabelecimento do neonato submetido à cirurgia cardíaca.

O presente estudo teve como objetivo caracterizar os neonatos em PO de cirurgia cardíaca, internados em um hospital privado do Município de São Paulo, referência em cirurgia cardíaca neonatal.

\section{MÉTODO}

Trata-se de um estudo transversal, com coleta retrospectiva de dados obtidos do registro de prontuário de neonatos submetidos a cirurgias cardíacas, com idade gestacional de nascimento igual ou superior a 35 semanas, em um hospital geral, privado, de médio porte, situado na zona oeste do Município de São Paulo, referência em cirurgia cardíaca neonatal. A referida instituição iniciou atendimento cirúrgico a neonatos com malformação cardíaca, a partir de julho de 2001.

Foram analisadas as informações referentes ao primeiro $\mathrm{PO}\left(1^{\circ} \mathrm{PO}\right)$ de RN submetidos à cirurgia cardíaca, paliativa ou corretiva, entre julho de 2001 e dezembro de 2005. Foram excluídos os prontuários de RN com outras malformações sistêmicas, além da cardíaca e aqueles com óbito do intraoperatório ao $1^{\circ} \mathrm{PO}$.

As seguintes variáveis foram coletadas e analisadas: idade gestacional de nascimento (em semanas completas), peso (em gramas) na data da cirurgia, idade pós-natal na data da cirurgia, diagnóstico(s) pré-opera-tório(s), tipo de intervenção e incisão cirúrgica e dispositivos invasivos em uso no $1^{\circ} \mathrm{PO}$.

Os prontuários digitalizados foram consultados eletronicamente e o acesso à base de dados foi feito na Instituição, campo do estudo. Os prontuários não localizados eletronicamente foram solicitados ao serviço de arquivo médico da instituição e os dados coletados dos prontuários impressos.

Estes foram registrados em instrumento de coleta elaborado para esse fim e armazenados em planilha Microsoft Excel for Windows XP. Posteriormente, foram transportados ao programa Epi-Info, versão 6.4 para análise estatística descritiva.

Antes do início da pesquisa, o projeto foi apreciado e aprovado pelo Comitê de Ética em Pesquisa da instituição, campo do estudo, sob n. 22/05.

\section{RESULTADOS}

Desde julho de 2001, quando a instituição passou a realizar cirurgias cardíacas em neonatos, até dezembro de 2005, 42 neonatos foram operados, dos quais, 12 não foram incluídos no estudo em razão dos critérios estabelecidos. Assim, 30 prontuários compuseram a casuística do presente estudo.

$O$ registro da idade gestacional ao nascimento foi identificado em 25 (83,4\%) prontuários, dos quais, três (12,0\%) $\mathrm{RN}$ eram pré-termos, com idade gestacional entre $35 \mathrm{se}$ manas e 36 semanas e $22(88,0 \%)$ neonatos eram a termo. A média da idade gestacional foi 37,64 e a mediana 38 , desvio-padrão de 1,15 semana. 
Quanto ao peso na data da cirurgia, nove (30,0\%) RN tinham entre 2.000 e 2.499 gramas; oito $(26,7 \%)$ RN pesavam entre 2.500 e 2.999 gramas; oito $(26,7 \%) \mathrm{RN}$ entre 3.000 e 3.499 gramas e cinco $(16,6 \%)$ pesavam 3.500 gramas ou mais. O peso dos RN variou de $2.010 \mathrm{~g}$ a 4.120 gramas. A média do peso foi de $2.885 \mathrm{~g}$ e a mediana, $2.837 \mathrm{~g}$, com desvio-padrão de $573 \mathrm{~g}$. 0 grupo também se mostrou homogêneo em relação ao peso na data da cirurgia.

A cirurgia cardíaca foi realizada nos primeiros seis dias de vida em 21 (70,0\%) neonatos e nove (30,0\%) RN foram operados no período neonatal tardio, do $7^{\circ}$ ao $28^{\circ}$ dia de vida. A idade dos neonatos na ocasião da cirurgia variou entre um e 25 dias de vida.

A média de idade do RN quando submetido à cirurgia cardíaca foi 6,5 dias, a mediana de 4 dias de vida e o desvio-padrão de 6,4 dias. Os dados mostram que as cirurgias cardíacas ocorreram precocemente, indicando a urgência com que foram realizadas as intervenções para garantir a sobrevida da criança.

Quanto às anomalias cardíacas congênitas, identificaram-se 24 diferentes diagnósticos, sendo consideradas todas as alterações anatômicas registradas nos prontuários médicos. Em seis $(20,0 \%)$ prontuários, havia registro de apenas um diagnóstico de anomalia cardíaca e um (3,3\%) RN apresentou oito alterações cardíacas registradas no prontuário médico. A média do número de anomalias cardíacas diagnosticadas foi 2,86 por RN.

A Tabela 1 mostra a distribuição dos RN, por diagnóstico de anomalia cardíaca.

Tabela 1 -Freqüência de RN por diagnóstico de anomalia cardíaca - São Paulo - 2001 - 2005

\begin{tabular}{lcc}
\hline Diagnóstico & n & \% \\
\hline Persistência do canal arterial & 21 & 70,0 \\
Comunicação interatrial & 12 & 40,0 \\
Transposição de grandes artérias & 11 & 36,3 \\
Defeitos valvares & 10 & 33,3 \\
Comunicação interventricular & 8 & 26,6 \\
Coarctação de aorta & 7 & 23,3 \\
Hipoplasia do coração esquerdo & 5 & 16,6 \\
Tetralogia de Fallot & 2 & 6,66 \\
Hipoplasia de ventrículo & 2 & 6,66 \\
Dupla via de saída de ventrículo & 2 & 6,66 \\
Atresia de valva pulmonar & 2 & 6,66 \\
Atresia de valva mitral & 2 & 6,66 \\
Outros * & 12 & 40,0
\end{tabular}

* As anomalias citadas, a seguir, foram identificadas em apenas um RN: Hipoplasia de arco aórtico, Dupla via de entrada de ventrículo único tipo esquerdo, Estreitamento de via de saída de ventrículo, Estenose de valva pulmonar, Valva pulmonar imperfurada, Valva aórtica bicúspede, Estenose de valva aórtica, Insuficiência de valva tricúspide, Ausência de veia cava superior esquerda, Bloqueio atrioventricular total, Drenagem anônima total de veias pulmonares, Isomerismo atrial esquerdo.
A persistência do canal arterial e comunicação interatrial foram as anomalias cardíacas mais freqüentes apresentadas pela população: 21 (70,0\%) RN e 12 (40,0\%) $\mathrm{RN}$, respectivamente.

Quanto ao procedimento cirúrgico, corretivo ou paliativo, identificou-se 14 diferentes procedimentos: sete $(23,3 \%)$ neonatos foram submetidos a apenas um tipo de procedimento, $12(40,0 \%)$ RN a dois tipos de intervenções, sete $(23,3 \%)$ RN a três tipos de intervenções e quatro $(13,4 \%)$ $\mathrm{RN}$ a quatro diferentes intervenções cirúrgicas. A média de procedimentos cirúrgicos realizados por RN foi de 2,26. A seguir, a Tabela 2 mostra a distribuição dos RN por procedimentos cirúrgicos realizados.

Tabela 2 - Freqüência de recém-nascidos por procedimento cirúrgico realizado - São Paulo - 2001 - 2005

\begin{tabular}{lcc}
\hline Procedimento cirúrgico & $\mathbf{n}$ & $\mathbf{\%}$ \\
\hline Ligadura do canal arterial & 19 & 63,3 \\
Anastomose de Blalock-Taussig & 10 & 33,3 \\
Correção de coarctação de aorta & 8 & 26,6 \\
Correção de comunicação interatrial ou interventricular & 7 & 29,1 \\
Atrioseptectomia ou atrioseptostomia & 7 & 29,1 \\
Bandagem do tronco pulmonar & 5 & 20,8 \\
Cirurgia de Jatene & 4 & 16,6 \\
Cirurgia de Norwood & 2 & 6,66 \\
Valvoplastia pulmonar & 2 & 6,66 \\
Outros* & 4 & 16,6 \\
\hline
\end{tabular}

* A seguir, os quatro procedimentos citados foram realizados em um RN: Correção de drenagem anômala de veias pulmonares, Anastomose tronco pulmonar e arco aórtico, Valvoplastia tricúspide, Implante de marcapasso interno.

Ligadura do canal arterial e anastomose BlalockTaussig foram os procedimentos cirúrgicos mais freqüentes encontrados nos registros dos prontuários, com 19 $(63,3 \%)$ e dez $(33,3 \%)$ RN, respectivamente.

Para a realização dos procedimentos, foram usados dois tipos de incisão: 22 (73,3\%) dos RN foram submetidos à incisão esternal e oito $(26,7 \%)$ neonatos, à incisão intercostal.

Os dispositivos invasivos em uso nos $\mathrm{RN}$, no $1^{\circ} \mathrm{PO}$, foram verificados em intervalos de seis horas, ou seja, entre as 24a e 29a horas, 30a e 35a horas, 36a e 41a horas e 42a e 47a horas.

A Figura 1 mostra os dispositivos: drenos de mediastino (DM) e pleural (DPL), sonda vesical de demora (SVD), cateter tenckoff (CT) e cânula traqueal (CTR) em uso nos RN.

A maioria dos RN, 26 (86,6\%), encontrava-se com cânula traqueal nas 24 a horas de PO. Já nas 47 a horas, houve redução do número de $\mathrm{RN}$ em ventilação pulmonar por meio de cânula orotraqueal, 21 (70,0\%) RN.

Uso de sonda vesical de demora foi verificado em 22 $(73,3 \%)$ neonatos nas 24 a horas de PO, nas 47 a horas, 21 $(70,0 \%)$ RN permaneciam com este dispositivo.

$\begin{array}{rr}\text { Rev Esc Enferm USP } & \text { Perfil de recém-nascidos submetidos à cirurgia cardíaca } \\ 2008 ; 42(1): 112-9 . & \text { em hospital privado do Município de São Paulo } \\ \text { www.ee.usp.br/reeusp/ } & \text { Bueno M, Kimura AF }\end{array}$




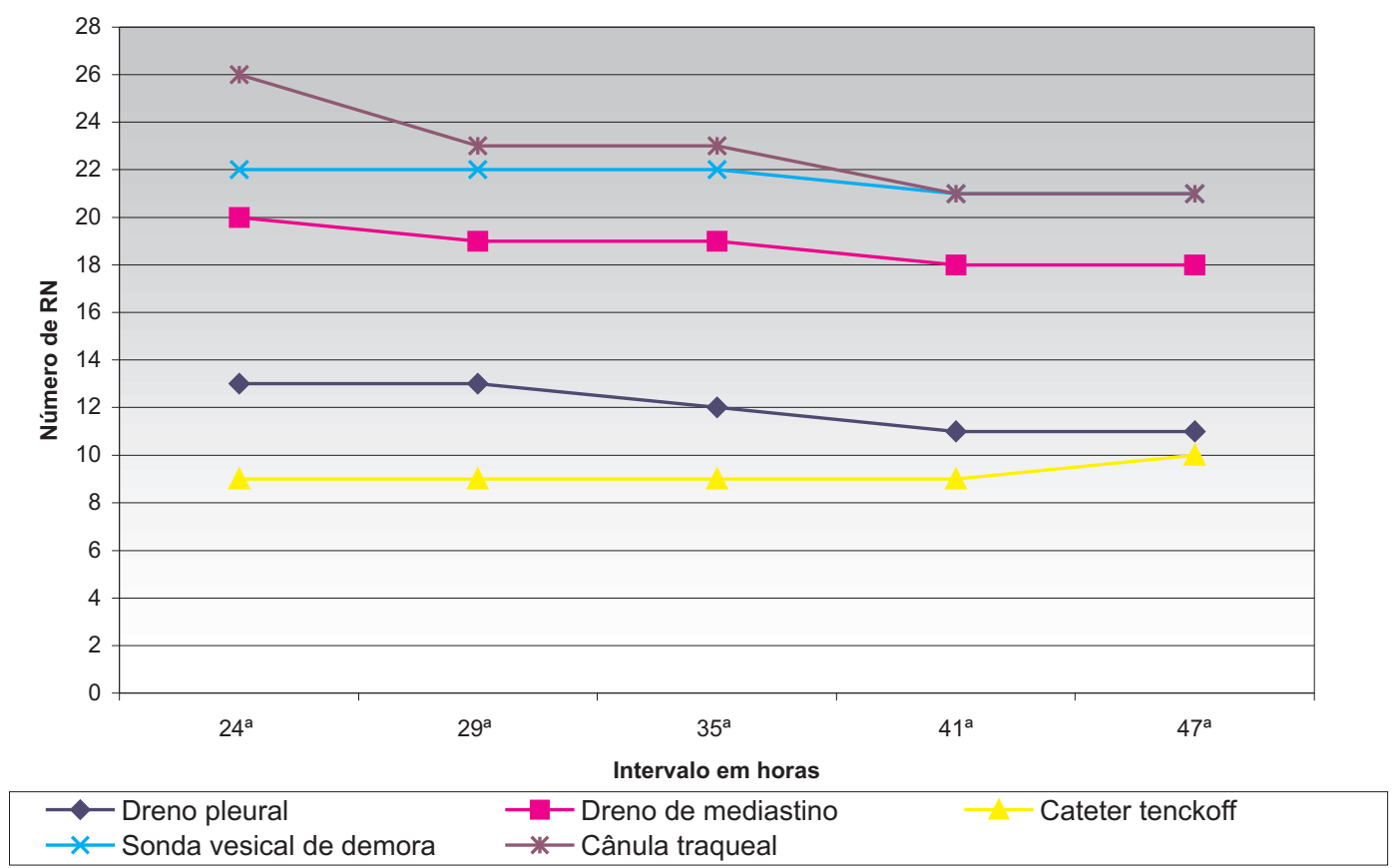

Figura 1 - Distribuição do número de RN, segundo dispositivos utilizados entre a $24^{\mathrm{a}}$ e $47^{\mathrm{a}}$ horas de pós-operatório - São Paulo - 2001 - 2005 Nota: n=30

O cateter tenckoff foi o único dispositivo cuja freqüência de uso aumentou de oito $(26,6 \%) \mathrm{RN}$, nas 24 a horas de $\mathrm{PO}$, para dez $(33,3 \%) \mathrm{RN}$, nas 47 a horas de $\mathrm{PO}$.

Além dos dispositivos apresentados na Figura 1, outros de acesso vascular foram instalados para monitoração dos parâmetros vitais e tratamento parenteral.
A Figura 2 apresenta os dispositivos vasculares: cateter venoso central (CVC), cateter central de inserção periférica (PICC), acesso venoso periférico (AVP), cateter umbilical venoso (CUV), cateter arterial e cateter de átrio direito (CAD) em uso nos RN no $1^{\circ} \mathrm{PO}$.

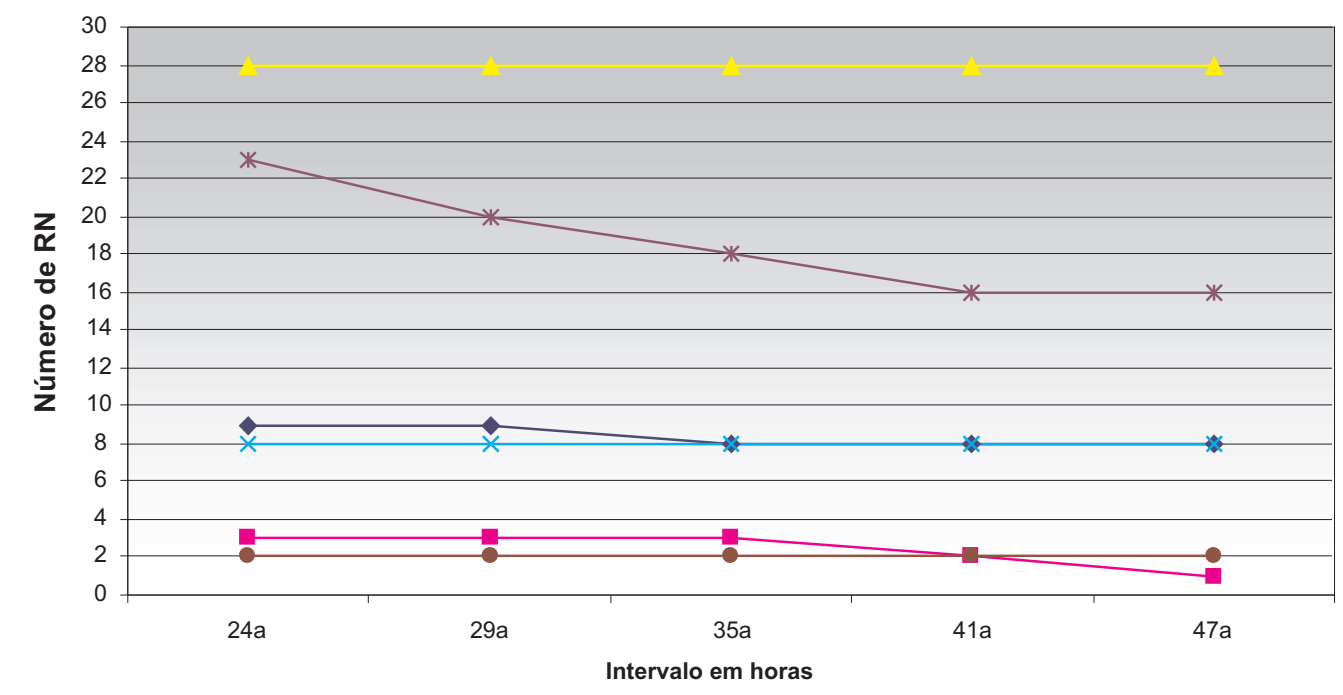

\begin{tabular}{ll|}
\hline- Acesso venoso periférico & - Cateter umbilical venoso \\
- Cateter venoso central & - Cateter central de inserção perfiérica \\
- Cateter arterial & - Cateter de átrio direito \\
\hline
\end{tabular}

Figura 2 - Distribuição do número de RN, segundo dispositivos vasculares utilizados entre a $24^{\mathrm{a}}$ e $47^{\mathrm{a}}$ horas de pós-operatório - São Paulo - 2001 - 2005 Nota: n=30 
Pela Figura 2, verifica-se que $28(93,3 \%)$ RN permaneciam com cateter venoso central no $1^{\circ} \mathrm{PO}$.

Nas 24a horas PO, 23 (76,6\%) RN estavam com cateter arterial e nas 47a horas, a freqüência de RN com este dispositivo reduziu para $16(53,4 \%)$ RN.

Dispositivos de acesso venoso periférico e cateter PICC foram usados em nove $(30,0 \%)$ RN e oito $(26,7 \%)$ RN respectivamente, conforme mostra a Figura 2.

\section{DISCUSSÃO}

Neonatos com idade gestacional entre 34 e 37 semanas ao nascimento, têm sido classificados como RN próximos do termo (near term) e não como prematuros ${ }^{(11)}$ por apresentarem desenvolvimento semelhante ao observado aos neonatos de termo quando comparados aos prétermos, com idade gestacional inferior a 34 semanas. Além disso, o peso de nascimento desses neonatos varia entre 2.000 e 2.500 gramas, em média, maior que o peso de nascimento de prematuros ${ }^{(11)}$.

Os dados deste estudo mostraram que $22(88,0 \%)$ neonatos nasceram com idade gestacional igual ou superior a 37 semanas e que $21(60,0 \%)$ RN tinham peso superior a 2.500 gramas, mostrando que, apesar da anomalia cardíaca congênita, não houve restrição do ganho ponderal intra-útero.

A idade gestacional e o baixo peso ao nascer são aspectos relevantes na abordagem ao RN cardiopata, consideradas como fatores de risco para avaliar o comprometimento neurológico e associadas à maior taxa de mortalidade precoce no $\mathrm{PO}^{(12-13)}$.

Embora $70,0 \%$ das cirurgias tenham sido realizadas na primeira semana de vida nos neonatos estudados, é recomendável que os procedimentos sejam feitos no período neonatal tardio, aguardando a adaptação orgânica da criança à vida extra-uterina ${ }^{(14)}$. Importante ressaltar que o restabelecimento, ainda no período neonatal, da função cardiovascular por meio de intervenção cirúrgica, promove efeitos benéficos na maturação de órgãos como os pulmões, o cérebro e o próprio coração ${ }^{(12)}$.

A intervenção cirúrgica realizada entre as $2 \underline{a}$ e a $3^{\underline{a}}$ semanas de vida é necessária nos neonatos, cujas cardiopatias são dependentes de fluxo sangüíneo, sistêmico e pulmonar pelo canal arterial como na tetralogia de Fallot, transposição das grandes artérias, drenagem anômala de veias pulmonares ${ }^{(7)}$. Além disso, RN portadores de cardiopatias com desvio intracardíaco de fluxo sangüíneo da esquerda para a direita, a exemplo do coração esquerdo hipoplásico, coarctação de aorta, estenose aórtica e interrupção do arco aórtico recomendam-se que as intervenções cirúrgicas sejam realizadas ainda no período neonatal ${ }^{(7)}$.

A precocidade das intervenções cirúrgicas, no período neonatal precoce, realizadas nos RN estudados justifica- se pela gravidade e complexidade das malformações diagnosticadas e de suas repercussões hemodinâmicas, com risco de expor o RN a complicações e morte.

Embora a população do estudo tenha sido constituída predominantemente de RN de termo, a alteração mais comum encontrada nos neonatos estudados foi a persistência do canal arterial, diagnosticada em 21 (70,0\%) neonatos.

Trata-se de uma patologia de importante repercussão clínica no prematuro, sendo sua incidência tanto maior quanto menor o peso corpóreo ocorrendo em até $70 \%$ dos neonatos com peso inferior a 1000 gramas $^{(15-16)}$. O tratamento pode ser farmacológico ou cirúrgico e não é uma patologia de significância clínica importante em RN de termo ${ }^{(4,8)}$.

Destaca-se que, embora grande parte da população estudada tenha sido de RN a termo, a presença da persistência do canal arterial esteve associada a outros defeitos cardíacos na maioria dos RN.

Os diagnósticos de 35 crianças com até quatro meses de idade submetidas a cirurgias cardíacas em uma instituição australiana ${ }^{(17)}$ incluíram: transposição de grandes artérias em 12 (34,3\%) RN; tetralogia de Fallot, em nove $(25,8 \%) \mathrm{RN}$; defeitos de septo ventricular, em oito $(22,8 \%)$ RN; drenagem anômala de vasos pulmonares, em dois $(5,7 \%) \mathrm{RN}$; truncus arteriosus, em dois $(5,7 \%) \mathrm{RN}$ e ventrículo único, em dois $(5,7 \%) \mathrm{RN}$.

Outro estudo recente ${ }^{(13)}$, registrou a seguinte incidência de defeitos cardíacos: $87(15,8 \%)$ crianças portadoras de defeito de septo ventricular; $54(9,8 \%)$ com transposição de grandes artérias; 53 (9,6\%) com tetralogia de Fallot; $53(9,6 \%)$ portadores de hipoplasia do coração esquerdo; $48(8,7 \%)$ apresentaram coarctação de aorta e $38(6,9 \%)$ com persistência do canal arterial.

Vale ressaltar que os estudos $\operatorname{citados}^{(13,17)}$ analisaram apenas o principal diagnóstico de anomalia cardíaca de cada neonato e não consideraram todas as malformações identificadas nos neonatos, como no presente estudo.

Isoladamente, a comunicação atrial é uma lesão simples, com incidência de $7 \%$ dentre os neonatos com anomalias cardíacas ${ }^{(16)}$ e taxa de mortalidade de $2 \%{ }^{(4)}$. Já a incidência de defeitos de septo ventricular, entre neonatos cardiopatas, varia entre 20 e $34,6 \%^{(18)}$ e com igual taxa de mortalidade $(2 \%)^{(4)}$.

Dentre as cardiopatias congênitas, a incidência de transposição de grandes artérias é de $7 \%^{(19)}$, mas quando não tratada cirurgicamente, a taxa de mortalidade na primeira semana de vida é de $28,7 \%$ e, no primeiro mês, chega a $56,1 \%{ }^{(20)}$.

A hipoplasia do coração esquerdo incide em 7\% a 9\% dos RN portadores de cardiopatias congênitas ${ }^{(5,21)}$. A taxa de mortalidade na primeira semana de vida é de até $25 \%{ }^{(21)}$, chegando a $95 \%$ em neonatos não submetidos a procedimento cirúrgico ${ }^{(5)}$. 
A incidência de coarctação de aorta é de $3,4 \%$ a $9,8 \%$ dentre as cardiopatias congênitas ${ }^{(16)}$. Dentre os pacientes não tratados cirurgicamente, a mortalidade é de $10 \%$ ainda no período neonatal|(22).

Tanto na literatura como na população estudada, foram verificadas incidências elevadas de defeitos de septo atrial e ventricular e de persistência do canal arterial, malformações relativamente simples. Os neonatos incluídos no estudo apresentaram tais malformações sempre associadas a outros tipos de lesões.

Quanto às malformações complexas, a transposição de grandes artérias, a hipoplasia do coração esquerdo e a coarctação de aorta foram as patologias mais prevalentes. Estas cardiopatias apresentam altas taxas de mortalidade, em especial, na primeira semana de vida. Os neonatos submetidos a cirurgias até o 60 dia de vida eram portadores de cardiopatias cuja anatomia do defeito cardíaco e as condições clínicas necessitavam de intervenções precoces.

Em relação aos procedimentos cirúrgicos, a correção da persistência do canal arterial (ligadura do canal) foi realizada em 19 (63,3\%) RN. É um procedimento relativamente simples e de baixa mortalidade quando realizado de modo isolado ${ }^{(4)}$. Entretanto, todos os neonatos do estudo submetidos à ligadura do canal, esta cirurgia foi realizada em associação com outras correções.

A anastomose de Blalock-Taussig foi a segunda intervenção cirúrgica mais freqüente, realizada em dez $(33,3 \%)$ RN. O procedimento consiste em implantar uma prótese para estabelecer comunicação entre as circulações sistêmica e pulmonar, a fim de melhorar o fluxo sangüíneo pulmonar ${ }^{(23-24)}$. Pode ser realizado isoladamente, como uma intervenção paliativa, em pacientes com diagnóstico de tetralogia de Fallot ou associado a outras intervenções cirúrgicas em pacientes com síndrome do coração esquerdo hipoplásico ${ }^{(4,24)}$.

A incisão esternal foi predominante, $22(73,3 \%) \mathrm{RN}$. Índice semelhante, $74 \%$, foi verificado em estudo ${ }^{(25)}$ realizado com crianças com até três meses de idade.

Todos os RN mantinham algum tipo de dispositivo invasivo para tratamento ou monitorização no $1^{\circ} \mathrm{PO}$.

Acesso venoso central, por punção ou venodissecção, necessário para a administração de drogas vasoativas, fluidos e hemoderivados e também para verificação da pressão venosa central no PO foi verificado em $27(90,0 \%)$ RN.

O uso da cânula traqueal, dispositivo imprescindível para manter adequada ventilação pulmonar no PO de cirurgias cardíacas neonatais foi verificado em 26 (86,6\%) RN. Alterações respiratórias no PO podem ocorrer devido à manutenção de circulação extra-corpórea intra-operatória e do nível de sedação no $\mathrm{PO}^{(1)}$.

Mantinham cateterização arterial, 23 (76,6\%) RN, necessária para a monitorização invasiva das pressões ar- teriais, sistólica, diastólica e média, no PO de cirurgia cardíaca ${ }^{(1,10)} \mathrm{e}$, também, é útil na coleta de amostras sangüíneas arteriais.

Os drenos, pleural e de mediastino estavam instalados em 13 (43,3\%) RN e 20 (66,6\%) RN, respectivamente, utilizados para remover as secreções resultantes da manipulação cirúrgica local, auxiliar na reexpansão pulmonar e remover qualquer quantidade de ar que possa evadir-se através de incisões realizadas na pleura ${ }^{(26-27)}$.

A função renal dos neonatos submetidos à cirurgia cardíaca pode ser afetada pela circulação extracorpórea, empregada durante a cirurgia, hipotermia, e ocorrência do baixo débito cardíaco, comum no pós-operatório ${ }^{(9)}$.

$O$ volume urinário reflete indiretamente o débito cardíaco e perfusão tissular ${ }^{(10)}$. Assim, o controle rigoroso de débito urinário, pela manutenção da sonda vesical de demora, é imprescindível no PO. Este dispositivo estava instalado em 22 (73,3\%) RN.

A diálise peritoneal é realizada no PO de cirurgia cardíaca neonatal para tratamento de insuficiência renal aguda $^{(28)}$, por meio do cateter tenckoff, geralmente, instalado durante a cirurgia, Verificou-se que nove $(30,0 \%)$ RN estavam do cateter tenckoff no 1 으. PO.

Outros dispositivos de acesso vascular foram verificados, com menor freqüência, na população estudada com a finalidade de administrar fluidos e fármacos. O cateter de átrio direito também é utilizado com o mesmo objetivo e para a monitorização de pressão de átrio direito.

A compatibilidade entre os fármacos prescritos e a velocidade com que esses fluidos e medicamentos são infundidos são fatores determinantes para o número de vias de acesso venoso ${ }^{(2)}$. No presente estudo, diversos tipos de dispositivos vasculares foram utilizados em razão do volume e da diversidade de fármacos prescritos.

Os dados referentes à caracterização dos RN do presente estudo possibilitam concluir que a assistência ao $\mathrm{RN}$ em pós-operatório de cirurgia cardíaca requer monitorização contínua dos parâmetros vitais em decorrência da incisão cirúrgica em si e dos diversos dispositivos instalados no RN.

\section{CONCLUSÃO}

A população estudada constituiu-se predominantemente de neonatos a termo e com peso superior a 2.500 gramas na data da cirurgia. A intervenção precoce, realizada ainda na primeira semana de vida, favorece a maturação de órgãos e sistemas prejudicados pela malformação cardíaca.

A persistência do canal arterial e os defeitos de septo foram os diagnósticos mais freqüentes na população estudada. Embora tais anomalias sejam consideradas de 
resolução simples e associadas a prognóstico favorável para sobrevivência dos $\mathrm{RN}$ a termo, estiveram associadas a outras malformações complexas.

A elevada prevalência de incisões esternais, 22 (73,3\%) $\mathrm{RN}$, indica a extensão do procedimento cirúrgico realizado nos RN.

Os inúmeros dispositivos em uso no $1^{\circ} \mathrm{PO}$ para controle dos parâmetros vitais, balanço hídrico, manutenção de suporte ventilatório, hemodinâmico, renal, além dos tratamentos realizados por acesso vascular refletem a instabilidade orgânica dos neonatos no PO de cirurgia cardíaca.

O conhecimento a respeito da anatomia dos defeitos cardíacos, das repercussões na circulação e oxigenação

\section{REFERÊNCIAS}

1. João PRD, Faria Junior A. Cuidados imediatos no pósoperatório de cirurgia cardíaca. J Pediatr (Rio). 2003;79 Supl 2:S213-22.

2. Miller-Hoover SR. Pediatric and neonatal cardiovascular pharmacology. Ped Nurs. 2003; 29(2):105-13.

3. Aylin P, Bottle A, Jarman B, Elliot P. Paediatric cardiac surgical mortality in England after Bristol: descriptive analysis of hospital episode statistics 1991-2002. BMJ. 2004; 329(7470):825-30.

4. Pedra CAC, Jatene MB, Riso A, Stape A. Pós-operatório de cirurgia cardíaca. In: Knobel E. Pediatria e neonatologia. São Paulo: Atheneu; 2005. p. 191-222.

5. Callow LB. Current strategies in the nursing care for infants with hipoplastic left-heart syndrome undergoing first-stage palliation with the Norwood operation. Heart Lung. 1992; 21(5):463-70.

6. Wechsler SB, Wernovsky G. Distúrbios cardíacos. In: Cloherty JP, Stark AR. Manual de neonatologia. Rio de Janeiro: MEDSI; 2000. p. 413-74.

7. Sharieff GQ, Wylie TW. Pediatric cardiac disorders. J Emerg Med. 2004;26(1):65-79.

8. Zanetta RAC, Afiune JY, Stape A. Abordagem das cardiopatias congênitas no recém-nascido. In: Knobel E. Pediatria e neonatologia. São Paulo: Atheneu; 2005. p.167-90.

9. Craig J. The postoperative cardiac infant: physiologic basis for neonatal nursing interventions. J Perinat Neonatal Nurs. 1991;5(2):60-70.

10.Guerra ALP. Cuidados pós-operatórios imediatos no recém-nascido. In: Santana MVT. Cardiopatias congênitas no recém-nascido: diagnóstico e tratamento. São Paulo: Atheneu; 2000. p. 401-9. neonatal, das intervenções cirúrgicas realizadas e dos dispositivos empregados no controle dos parâmetros vitais e no tratamento instituído a estes pacientes fundamentam uma adequada assistência.

O neonato submetido à cirurgia cardíaca é um paciente complexo que requer equipe de enfermagem devidamente capacitada para assisti-lo, com competência para prever e detectar complicações precoces e atuar em seu pronto restabelecimento.

Nesse sentido, conhecer o perfil da população assistida é fundamental aos gestores e profissionais que atuam no serviço de saúde, já que estas informações subsidiam o planejamento dos recursos humanos, materiais e logísticos necessários para prover uma assistência adequada ao tipo de população atendida.

11. Medoff-Cooper B, Bakewell-Sachs S, Bus-Frank ME, Santa-Donato A. The AWHONN Neart-Term Infant Initiative: a conceptual framework for optimizing health for nearterm infants. J Obstet Gynecol Neonatal Nurs. 2005;34(6):666-71.

12. Bové $T$, François $K$, De Groote $K$, Suys $B$, De Wolf $D$, Verhaaren $H$, et al. Outcome analysis of major cardiac operations in low weight neonates. Ann Thorac Surg. 2004;78(1): 181-7.

13. Parkman SE, Woods SL. Infants who have undergone cardiac surgery: what can we learn about lengths of stay in the hospital and presence of complications? J Pediatr Nurs. 2005; 20(6):430-40.

14. Fragata J. Neonatal cardiac surgery. Rev Port Cardiol. 2004;23(2):283-92.

15. Chaccur P, Dancini JL. Persistência do canal arterial. Cardiopatias congênitas no recém-nascido: diagnóstico e tratamento. In: Santana MVT. Cardiopatias congênitas no recém-nascido: diagnóstico e tratamento. São Paulo: Atheneu; 2000. p. 267-74.

16. Mesquita SMF, Ikari NM, Ebaid M. Cardiopatias congênitas acianogênicas. In: Ebaid $M$, coordendor. Cardiologia em pediatria: temas fundamentais. São Paulo: Rocca; 2000. p. 257-86.

17. Robertson DR, Justo RN, Burke CJ, Pohlner PG, Graham $\mathrm{PL}$, Colditz PB. Perioperative predictors of developmental outcome following cardiac surgery in infancy. Cardiol Young. 2004;14(4):389-95.

18. Silva CMC. Comunicação interventricular em neonato. In: Santana MVT. Cardiopatias congênitas no recémnascido: diagnóstico e tratamento. São Paulo: Atheneu; 2000. p. 251-66. 
19. Bustamante LN. Cardiopatias congênitas cianogênicas. In: Ebaid M, coordenador. Cardiologia em pediatria: temas fundamentais. São Paulo: Rocca; 2000. p. 287330.

20. Bosiro IBJ, Jatene $M$, Jatene A. Transposição das grandes artérias. In: Santana MVT. Cardiopatias congênitas no recém-nascido: diagnóstico e tratamento. São Paulo: Atheneu; 2000. p. 133-46.

21. Barbero-Maciel M, Tanamati C. Síndrome de hipoplasia do coração esquerdo. In: Santana MVT. Cardiopatias congênitas no recém-nascido: diagnóstico e tratamento. São Paulo: Atheneu; 2000. p. 123-32.

22. Fontes VF, Pedra CAC, Pedra SRF. Coarctação da aorta. In: Santana MVT. Cardiopatias congênitas no recém-nascido: diagnóstico e tratamento. São Paulo: Atheneu; 2000. p. 287-95.

23. Sabri MR, Sholler G, Hawker R, Nunn G. Branch pulmonary artery growth after blalock-taussig shunts in tetrallogy of Fallot and pulmonary atresia with ventricular septal defect: a retrospective, echocar-giographic study. Pediatr Cardiol. 1999;20(5):358-63.
24. Mongale P. Thrombosis in children with BT shunts, glenns and fontans. Prog Pediatr Cardiol. 2005;21 (1):17-21.

25. McRae ME, Rourke DA, Imperial-Perez FA. Development of a research-based standard for assessment, intervention, and evaluation of pain after neonatal and pediatric cardiac surgery. Ped Nurs. 1997;23(3):263-71.

26. Mueller XM, Tinguely F, Tevaearai HT, Ravussin P, Stumpe $S$, Von Segesser LK. Impact of duration of chest tube drainage on pain after cardiac surgery. Eur J Cardiothorac Surg. 2000;18(5):570-4.

27. Waldhausen JHT, Cusick RA, Graham DD, Pittinger TP, Sawim RS. Removal of chest tubes in children without water seal after elective thoracic procedures: a randomized prospective study. J Am Coll Surg. 2002; 194 (4):411-5.

28. Dittrich S, Vogel M, Dähnert I, Haas NA, Alexi-Meskishvili $\mathrm{V}$, Landge PE. Acute hemodynamic effects of post cardiotomy peritoneal dialysis in neonates and infants. Intensive Care Med. 2000;26(1):101-4. 